\title{
Analysis of Brillouin-Based Distributed Fiber Sensors Using Optical Pulse Coding
}

\author{
Prasant K. Sahu ${ }^{1}$, Marcelo A. Soto ${ }^{2}$, Jeonghwan Lee ${ }^{3}$, \\ Gabriele Bolognini' ${ }^{2}$, Namkyoo Park ${ }^{3}$, Fabrizio Di Pasquale ${ }^{2}$ \\ ${ }^{1}$ Dept. of Electronics \& Electrical Communication Engineering, Indian Institute of Technology, Kharagpur, 721302, India \\ ${ }^{2}$ Scuola Superiore Sant'Anna, via G. Moruzzi 1, 56124 Pisa, Italy.e-mail: fabrizio.dipasquale@sssup.it \\ ${ }^{3}$ School of EECS, Seoul National University, Korea.e-mail: nkpark@plaza.snu.ac.kr
}

\begin{abstract}
We propose the use of pulse Simplex-coding in distributed temperature sensors based on spontaneous Brillouin scattering. The analysis shows achieved performance improvement and the effect on stimulated Brillouin threshold, resulting in lower required input power.

C2008 Optical Society of America

OCIS codes: (060. 2370) Fiber optics sensors, (290.5830) Scattering, Brillouin.
\end{abstract}

\section{Introduction}

Fiber optic distributed sensors are becoming increasingly attractive for temperature and strain monitoring over short, medium and long-distance applications. In particular, the main advantage of fiber-based distributed temperature sensors, compared to conventional or point sensors, mainly lies in their unparalleled ability to continuously and accurately monitor the temperature over long sensing distances with high spatial resolution. Compared to the most commercially successful solution based on spontaneous Raman scattering, Brillouin based distributed sensors offer the attractive possibility to monitor both temperature and strain simultaneously [1]; in particular, Brillouin-based distributed temperature sensors (BDTS) are characterized by higher backscattering intensities and lower temperature sensitivity compared to Raman-based DTS, but they also require a more complex receiver structure. BDTS can be implemented using either spontaneous or stimulated Brillouin scattering; while spontaneous-BDTS require simpler implementation schemes [1], stimulated-BDTS allows for higher accuracy and resolution in the measurement but also requires access to both fiber ends and more complex detection [2].

In this paper we focus our attention on spontaneous Brillouin-based distributed temperature sensors using coded optical time domain reflectometry (OTDR) and a direct-detection scheme. In particular we experimentally show that Simplex coding [3], already applied for enhancing Raman-based DTS performance [4], provides a significant receiver signal-to-noise ratio (SNR) improvement, allowing for improved temperature/spatial resolution with respect to single pulse operation. The analysis also shows the effect of pulse coding on stimulated Brillouin scattering (SBS) threshold, resulting in lower optimum input power levels compared to single pulse case, avoiding the use of optical pulse amplification. The implemented sensor, based on the Landau-Placzek ratio (LPR) scheme [5], allows for temperature sensing over $21 \mathrm{~km}$ of dispersion shifted fiber (DSF) with $3 \mathrm{~K}$ temperature resolution (spatial resolution $40 \mathrm{~m}$ ) and $10 \mathrm{~mW}$ optical peak power launched into the sensing fiber, practically avoiding the use of Erbium-doped fiber amplifiers (EDFA).

\section{Theory}

The implemented BDTS is based on coded OTDR and uses the LPR scheme for temperature derivation. The LPR is defined as the ratio of intensities of Rayleigh scattering light $\left(I_{R S}\right)$ to the spontaneous Brillouin scattering component $\left(I_{B S}\right)$ [5], whose temperature dependence is given by the relation: $I_{R S} / I_{B S}=T_{f}\left(\beta_{T} \rho_{0} v_{s}^{2}-1\right) / T$, where $\beta_{T}$ is the fiber glass isothermal compressibility, $\rho_{0}$ the density, $T_{f}$ the fictive temperature and $v_{\mathrm{s}}$ the acoustic velocity. For actual measurements, the temperature estimation is obtained comparing the LPR at temperature $T$ with the LPR measured at a reference temperature $T_{R}$, giving:

$$
T=\frac{1}{K_{T}}\left(1-\frac{L P R(T)}{\operatorname{LPR}\left(T_{R}\right)}\right)+T_{R}
$$

where $K_{T}$ is the temperature sensitivity [6].

If detrimental nonlinear effects are negligible along the sensing fiber, the temperature resolution is essentially related to the noise level at the receiver and to the input pulse duration, which also determines the spatial resolution. In such a condition, pulse coding can potentially overcome the trade-off between sensing distance and resolution, providing significant SNR enhancement compared to conventional single pulses, as prescribed from coding gain, $G_{C O D}$, which for an arbitrary code length $L$ is given by [7]: $G_{C O D}=(L+1) /(2 \sqrt{ } L)$.

Actually, Simplex codes have been demonstrated to be efficient among other suitable coding schemes for applications such as OTDR and Raman-based sensors [4], allowing for a good improvement in SNR even at 


\section{OMT1.pdf}

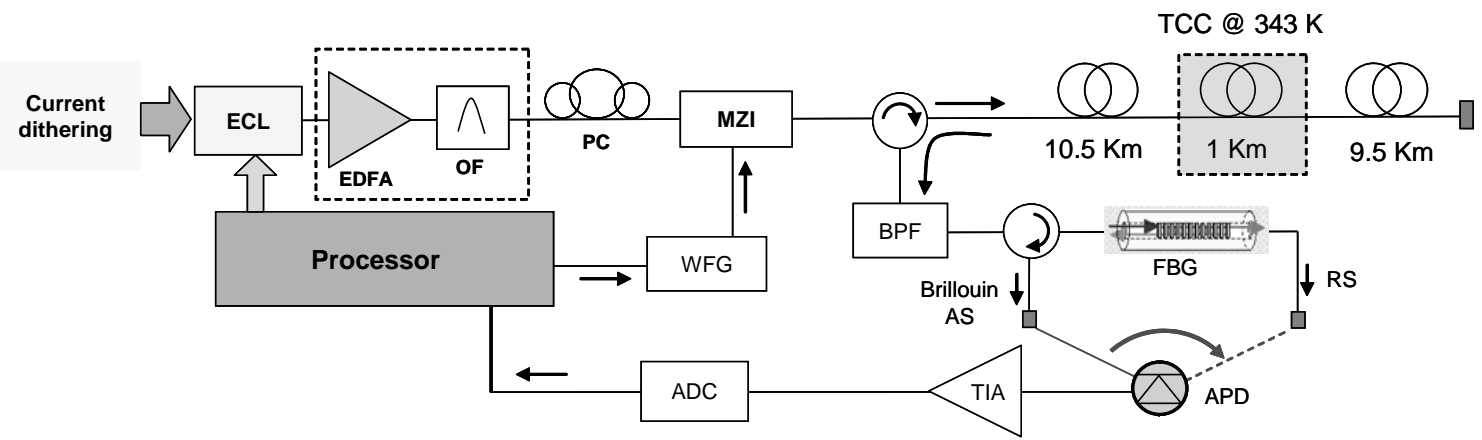

Fig. 1. Experimental setup to investigate the effects of pulse coding on spontaneous-BDTS performance.

short code lengths [7], allowing a more accurate spatial resolution and reducing the input pulse power level [4], with negligible influence on degrading nonlinear effects (such as stimulated Raman scattering).

However, the effectiveness of pulse coding applied to Brillouin-based sensors is not straightforward, since Brillouin effect has very different physical properties from Raman scattering. For instance, the narrower gain bandwidth, the counter-propagating direction of scattering light and the required use of narrowband sources in case of Brillouin-based sensors can have a strong impact on the effectiveness of coding techniques in spontaneous BDTS. Actually, coded pulses can in this case greatly reduce the threshold for onset of stimulated Brillouin scattering, while pulse coding used for Raman-based sensors [4] have shown a negligible impact on the threshold for stimulated Raman scattering (which is the most limiting effect in that case).

In this paper we show that using optical pulse coding in spontaneous BDTS can provide an effective SNR enhancement. We also observed that, since coded pulses strongly impact on stimulated Brillouin threshold power, they have significantly lower optimum input peak power levels than single pulses, hence providing accurate temperature measurements over long distances without using optical amplifiers.

\section{Experimental set-up}

The experimental set-up used to study spontaneous Brillouin based DTS using S-coding is reported in Fig. 1; the used source is a tunable external cavity laser (ECL) with a linewidth of $200 \mathrm{kHz}$ operating at $1550.1 \mathrm{~nm}$, and amplified by an EDFA. A Mach-Zehnder modulator (MZI), driven by a waveform generator (WFG), provides either single pulses or Simplex-coded pulses (400 ns pulse duration, ensuring a spatial resolution of $40 \mathrm{~m}$ ). A tunable optical bandpass filter (OF, $0.8 \mathrm{~nm}$ FWHM) is used immediately after the EDFA to reduce the amplified spontaneous emission (ASE) noise. The sensing fiber consists of three spools $(10.5 \mathrm{~km}, 1 \mathrm{~km}$, and $9.5 \mathrm{~km})$ of dispersion-shifted fiber (DSF) for a total length of $21 \mathrm{~km}$.

The modulated output light from the MZI is coupled into the sensing fiber through a three-port optical circulator. The middle fiber spool (length: $1 \mathrm{~km}$ ) is placed inside a temperature-controlled chamber (TCC), which allows for a precise control of the fiber temperature. Note that current dithering has been applied to the laser source in order to broaden its linewidth, hence increasing the power threshold value before the onset of detrimental stimulated Brillouin scattering, and also helping to mitigate Coherent Rayleigh Noise (CRN) in OTDR traces. In order to further reduce the CRN in Rayleigh-scattered light measurements, we have also applied wavelength averaging technique ( $60 \mathrm{GHz} \lambda$-sweeping of ECL).

The receiver section consists of a three-port optical circulator, a narrowband fiber Bragg grating (FBG, 6 $\mathrm{GHz}$ bandwidth at anti-Stokes port), a high gain InGaAs avalanche photodiode (APD, $80 \mathrm{MHz}$ ) followed by a high gain transimpedance amplifier (TIA) and by an analog-digital converter (ADC). The FBG is used to separate the Anti-Stokes Brillouin-scattered light from the Rayleigh-scattered light ( $28 \mathrm{~dB}$ band rejection) into two different circulator ports.



Fig. 2. Temperature-dependent Anti-Stokes trace with conventional and Simplex-coded DTS.

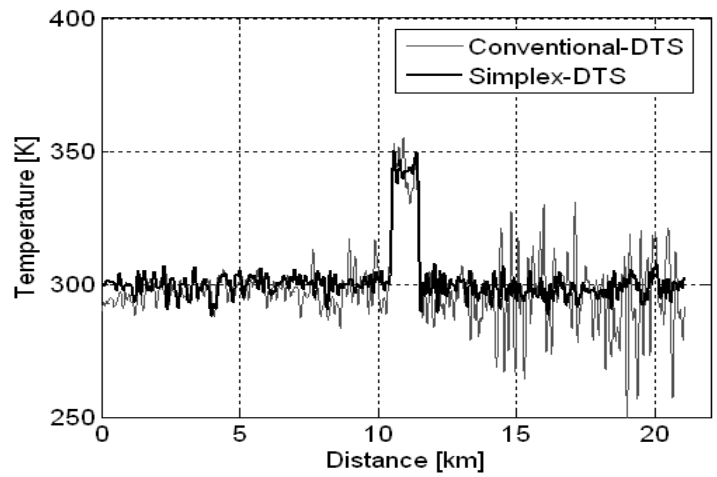

Fig. 3. Temperature distribution along the fiber obtained from LPR for conventional and Simplex-coded DTS 


\section{OMT1.pdf}

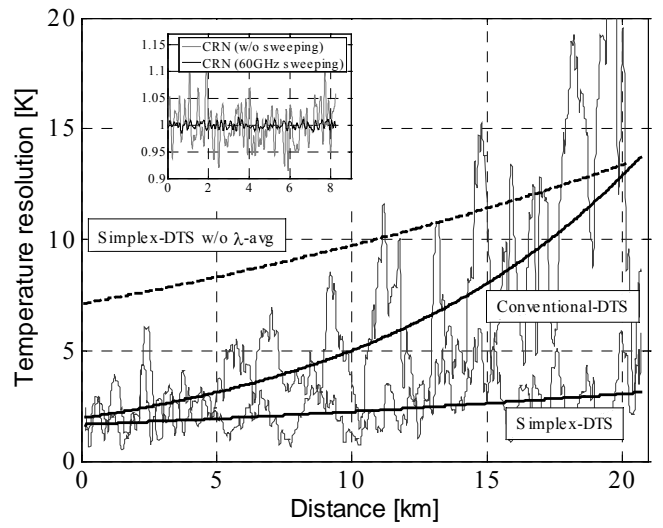

Fig. 4. Temperature resolution versus fiber length (conventional-DTS, Simplex-DTS with and without $\lambda$-averaging)

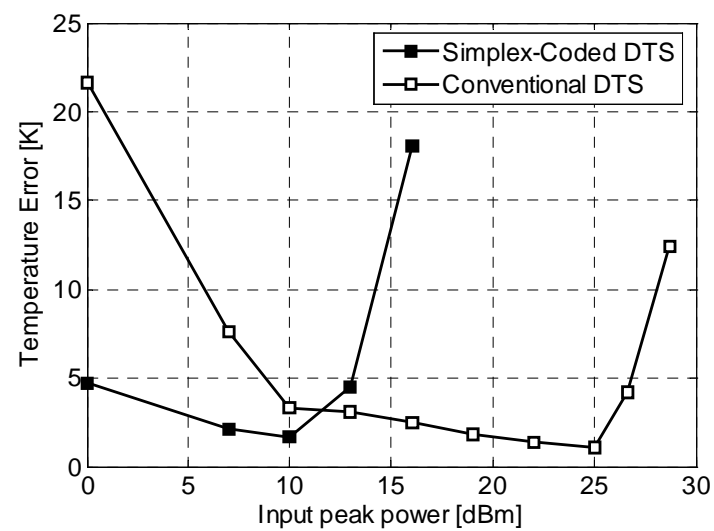

Fig. 5. Temperature resolution (at $4 \mathrm{~km}$ distance) versus input peak power conditions for conventional and Simplex-DTS

\section{Results and discussion}

The temperature along the sensing fiber has been derived using Eq. (1), where the reference temperature was considered to be the room temperature $(300 \mathrm{~K})$. Fig. 2 shows the temperature dependence of the anti-Stokes trace along fiber length when the TCC is set to $343 \mathrm{~K}$, Fig. 3 shows the calculated temperature distribution along fiber length; the noise reduction using Simplex coding is clearly evident from both figures. The SNR enhancement has a strong impact on the attained temperature resolution (computed by fitting the rms of the difference between estimated and real temperature) as shown in Fig. 4. The measured SNR improvement $(7.12 \mathrm{~dB})$ in LPR using 127-bit Simplex coding with respect to single pulse case is in good agreement with theory $(7.54 \mathrm{~dB})$; the total number of acquired traces is $180 \mathrm{k}$ in both cases. Using $10 \mathrm{dBm}$ input signal power and $400 \mathrm{~ns}$ pulses, we can hence achieve (see Fig. 4) a temperature resolution near the fiber end (21 km) equal to $13.7 \mathrm{~K}$ with single pulses, improving to $3.1 \mathrm{~K}$ with the use of Simplex coding. Note that current dithering and wavelength averaging play a key role in reducing CRN in $I_{R S}$ trace. Fig. 4 clearly shows the effects of wavelength averaging on the temperature resolution (a significant $\mathrm{CRN}$ reduction from $2.6 \%$ to $0.4 \%$ is obtained in $I_{R S}$ trace, shown in Fig. 4 inset). Fig. 4 also highlights the serious impact of CRN on temperature resolution; actually, the Simplex-coded DTS scheme without $\lambda$-averaging (dotted line) provides a worse temperature resolution than conventional-DTS scheme with $\lambda$-averaging, especially near fiber input.

Finally, Fig. 5 compares the obtained temperature resolution (at $4 \mathrm{~km}$ distance) with conventional-BDTS and Simplex-BDTS versus launch peak power. It can be seen from Fig. 5 that the temperature estimation accuracy starts to be degraded for input power level above the SBS threshold value in both cases. However, very different power levels corresponding to SBS onset are observed with coding and with single pulses. This can limit the ability of pulse coding to achieve an overall better temperature resolution than single pulse case, differently from Raman sensor case where this effect was not observed [4]. This can be understood since the pulse coding process leads to a longer effective pulse length, and consequently to a strongly decreased SBS threshold power, limiting the usable power level. Hence the optimal resolution with Simplex-DTS (attaining similar values to single pulse case) is achieved at much lower power level with respect to conventional-DTS (about $15 \mathrm{~dB}$ lower power from Fig. 5), allowing one to avoid optical pulse amplification.

In conclusion, we have proposed and experimentally characterized the behaviour of Simplex-coding technique in BDTS using LPR for temperature resolution enhancement. Simplex-coding technique, although having a non-negligible impact on stimulated Brillouin scattering threshold power, allows for a temperature resolution similar to single pulse case at significantly lower input peak power. Coding techniques can then be successfully applied as an alternative to optical pulse amplification in BDTS, providing a high-performance cost-effective solution.

\section{References}

[1] S. M. Maughan et al., "Simultaneous distributed fiber temperature and strain sensor using microwave coherent detection of spontaneous Brillouin backscatter," Meas. Sci. Technol., vol. 12, pp. 834-842 (2001).

[2] A. Minardo et al, "Accurate distributed temperature measurements by Brillouin Scattering fiber-optic sensor," Proceedings of IEEE Sensors Conference, vol. 1, pp. 348- 352 (2003).

[3] M. D. Jones, "Using Simplex codes to improve OTDR Sensitivity," IEEE Photon. Technol. Lett., vol. 15, pp. 822-824 (1993).

[4] G. Bolognini et al., "Performance enhancement of Raman-based distributed temperature sensors using simplex codes," OFC Conf. Digest, OSA, Mar 2006.

[5] P. C. Wait et al., "Landau Placzek ratio applied to distributed fiber sensing," Opt. Commun., vol. 122, pp. 141-146 (1996).

[6] K. De. Souza et al, "Improvement of signal-to-noise capabilities of a distributed temperature sensor using optical preamplification," Meas. Sci. Technol., vol.12, pp. 952- 957 (2001).

[7] D. Lee et al, "Analysis and experimental demonstration of simplex coding technique for SNR enhancement of OTDR," LTIMC-15, Proceedings IEEE LTIMC, New York, Oct 2004. 